\title{
Temporally shaping biphoton wave packets with periodically modulated driving fields
}

\author{
Shengwang Du, ${ }^{1, *}$ Jianming Wen, ${ }^{2}$ and Chinmay Belthangady ${ }^{3}$ \\ ${ }^{1}$ Department of Physics, The Hong Kong University of Science and Technology, Clear Water Bay, Kowloon, Hong Kong, China \\ ${ }^{2}$ Department of Physics, University of Virginia, Charlottesville, Virginia 22904, USA \\ ${ }^{3}$ Edward L. Ginzton Laboratory, Stanford University, Stanford, California 94305, USA
}

(Received 17 November 2008; revised manuscript received 13 January 2009; published 10 April 2009)

\begin{abstract}
We describe a method for waveform shaping of narrowband biphotons generated in a double- $\Lambda$ atomic system via four-wave mixing by periodically modulating the input driving lasers. We show that the input field profile(s) can be revealed in the two-photon temporal waveform and its correlation measurement.
\end{abstract}

DOI: 10.1103/PhysRevA.79.043811

PACS number(s): 42.50.Dv, 32.80.Qk, 42.50.Gy, 42.65.Lm

\section{INTRODUCTION}

Generation of a quantum system with controllable wave functions is always a great interest to both fundamental and practical researches. For single photons, it has been demonstrated that their quantum waveforms can be precisely manipulated by external modulations $[1,2]$. For entangled paired photons, termed as biphotons, shaping their temporal profiles with arbitrary waveforms still remains a challenge. Conventionally, biphotons produced from spontaneous parametric down conversion (SPDC) from a nonlinear crystal are associated with broad bandwidth, typically in the terahertz range. Shaping of SPDC photons temporal wave function was achieved by performing spectral manipulations of the joint spectrum [3-5] or spatial modulation of the nonlinear interaction [6-8]. Effects of input pump pulse on the two-photon correlation and entanglement have been theoretically studied in Refs. $[9,10]$.

Recently, narrow-band biphotons generated from fourwave mixing (FWM) in both cold and hot atomic ensembles have attracted considerable interest because of their long coherence time and high spectral brightness [11-15]. Nonlocal modulation of temporal correlation has been proposed and observed with narrow-band biphotons [16,17]. At high optical depth (OD), subnatural linewidth biphotons [18] were generated with the assistance of slow light and electromagnetically induced transparency (EIT) [19]. Such a sufficiently long coherence time $(\sim 1 \mu \mathrm{s})$ may allow one to further modify biphoton wave packets directly in the time domain. However, up to this day waveform controlling of narrowband biphotons has only been performed in spectral domain through Rabi splitting [11,13,14], phase matching [18], or using optical cavities [12].

In this paper, we propose a method for manipulating biphoton quantum waveform in an EIT-based double- $\Lambda$ atomic system by periodically modulating two input driving fields. We show that at a high OD, the field-amplitude phase correlation between two input beams can be mapped into biphoton wave packet within its coherence time window. We note that the difference between the current method and the case with traditional SPDC is that the latter case deals with only one input pump beam, whose field profile indeed has an impact

\footnotetext{
*dusw@ust.hk
}

on the properties of nonclassical correlation and quantum entanglement but cannot be mapped into the biphoton waveform $[9,10]$. One advantage of using the current method is that it provides a flexible way to shape the two-photon wave function by manipulating two input pump beams. The twophoton correlation with continuous-wave (cw) driving lasers has been theoretically analyzed in Refs. [20-24] considering a four-level double- $\Lambda$ EIT system. However, because of the breakdown of the time translation symmetry, these methods might not be directly applicable to the time-varying driving field case, as shall be discussed here. Moreover, because of the modulations the EIT linear and nonlinear optical responses are generally different from the cw case.

We organize this paper as follows. In Sec. II we develop the theory of the two-photon optics using perturbation theory in the Schrödinger picture. In Sec. III we present the numerical simulations considering feasible experimental parameters. In Sec. IV we extend the theory to the case in which the pump and coupling fields have a continuous spectrum. Finally, we draw our conclusion in Sec. V. The results presented here may be of importance for applications in quantum communication, quantum information processing, and quantum state engineering. Generating entangled photon pairs on demand can be envisioned in this method.

\section{THEORY}

We illustrate the basic idea in Fig. 1. As shown in Fig. $1(\mathrm{a})$, in the presence of counterpropagating pump $\left(\omega_{p}\right)$ and coupling $\left(\omega_{c}\right)$ laser beams, backward-propagating Stokes

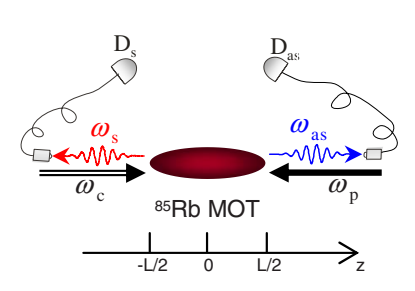

(a)

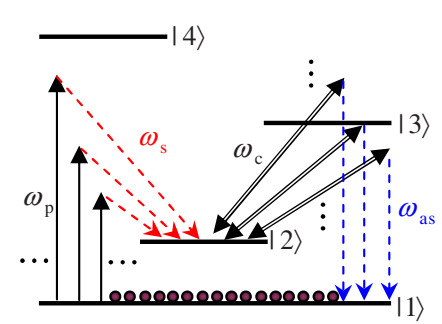

(b)
FIG. 1. (Color online) Schematic of biphoton generation with periodically modulated driving-laser fields. (a) Experimental backward configuration. (b) Multichannel biphoton generation via fourwave mixing in a double- $\Lambda$ atomic system. 
$\left(\omega_{s}\right)$ and anti-Stokes $\left(\omega_{a s}\right)$ photon pairs are spontaneously generated from a cold atomic ensemble into opposing singlemode fibers and detected by single-photon counting modules, $D_{s}$ and $D_{a s}$, respectively. Periodically modulated pump and coupling laser fields are decomposed into discrete frequency components as depicted in Fig. 1(b). We will show that the interference between biphotons generated from different-channel FWMs provides a controllable way to modify their wave packets in the time-frequency domain.

We develop the theory in the Schrödinger picture and take the plane-wave approximation for all involved optical fields. Within the medium the periodically modulated pump and coupling fields at positive frequencies are represented by their discrete Fourier series, that is,

$$
\begin{gathered}
E_{p}^{(+)}(z, t)=\sum_{m} E_{p m} e^{i\left(-k_{p m} z-\omega_{p m} t\right)}, \\
E_{c}^{(+)}(z, t)=\sum_{n} E_{c n} e^{i\left(k_{c n} z-\omega_{c n} t\right)},
\end{gathered}
$$

where $k_{p m, c n}$ are wave numbers of input pump fields with discrete Fourier frequency components $\omega_{p m, c n}$, and $E_{p m, c n}$ are their corresponding field-amplitude profiles, respectively. The single-transverse-mode Stokes and anti-Stokes fields within the system are taken as quantized field operators,

$$
\hat{E}_{s}^{(+)}(z, t)=\frac{1}{\sqrt{2 \pi}} \sum_{m} \sqrt{\frac{2 \hbar \omega_{s m}}{c \varepsilon_{0} A}} \int d \omega \hat{a}_{s m}(\omega) e^{i\left[-k_{s m}(\omega) z-\left(\omega_{s m}+\omega\right) t\right]},
$$

$$
\hat{E}_{a s}^{(+)}(z, t)=\frac{1}{\sqrt{2 \pi}} \sum_{n} \sqrt{\frac{2 \hbar \omega_{a s n}}{c \varepsilon_{0} A}} \int d \omega \hat{a}_{a s n}(\omega) e^{i\left[k_{a s n}(\omega) z-\left(\omega_{a s n}+\omega\right) t\right]},
$$

with the single-mode cross-section area $A . \omega_{s m}$ and $\omega_{a s n}$ are central angular frequencies of Stokes and anti-Stokes photons at channel $(m, n)$, respectively, and satisfy the frequency matching condition $\omega_{p m}+\omega_{c m}=\omega_{s m}+\omega_{a s m}$. The annihilation field operators $\hat{a}_{s m}(\omega)$ and $\hat{a}_{a s n}(\omega)$ satisfy the commutation relation, $\left[\hat{a}_{s m}(\omega), \hat{a}_{s n}^{\dagger}\left(\omega^{\prime}\right)\right]=\left[\hat{a}_{a s m}(\omega), \hat{a}_{a s n}^{\dagger}\left(\omega^{\prime}\right)\right]$ $=\delta_{m n} \delta\left(\omega-\omega^{\prime}\right)$.

In the interaction picture, the Hamiltonian takes the form $\hat{H}_{I}(t)=\frac{\varepsilon_{0} A}{4} \int d z \chi^{(3)} E_{c}^{(+)} E_{p}^{(+)} \hat{E}_{s}^{(-)} \hat{E}_{a s}^{(-)}+$H.c. [20], where $\hat{E}_{s, a s}^{(-)}$is the Hermitian conjugate (H.c.) of $\hat{E}_{s, a s}^{(+)}$and $\chi^{(3)}$ is the thirdorder nonlinear susceptibility. For simplicity, we assume that both the pump and coupling frequency spacings are at least comparable with or larger than the bandwidth of each FWM channel such that there is no spectral overlap between the different FWM channels, as schematically shown in Fig. 1(b). With the use of Eqs. (1)-(4), the interaction Hamiltonian becomes

$$
\begin{aligned}
\hat{H}_{I}(t)= & \frac{i \hbar L}{2 \pi} \sum_{m, n} \int d \omega^{\prime} \int d \omega \operatorname{sinc}\left(\frac{\Delta k_{m n} L}{2}\right) \kappa_{m n}\left(\omega^{\prime}, \omega\right) \\
& \times E_{p m} E_{c n} \hat{a}_{s m}^{\dagger}\left(\omega^{\prime}\right) \hat{a}_{a s n}^{\dagger}(\omega) e^{i\left(\omega^{\prime}+\omega\right) t}+\text { H.c. }
\end{aligned}
$$

where $\Delta k_{m n}\left(\omega^{\prime}, \omega\right)=k_{a s n}(\omega)-k_{s m}\left(\omega^{\prime}\right)-k_{c n}+k_{p m}$ is the phase mismatching of the $(m, n)$ th FWM channel, $\kappa_{m n}\left(\omega^{\prime}, \omega\right)$ $=-i \frac{\sqrt{\omega_{s m} \omega_{a s n}}}{2 c} \chi_{m n}^{(3)}\left(\omega^{\prime}, \omega\right)$ represents the nonlinear coupling coefficients, and $L$ is the length of the medium. The sinc function in Eq. (5) comes from completing the integral over the interaction length.

Using the perturbation theory $[20,25]$, we obtain the photon state approximately as a linear superposition of the vacuum state $|0\rangle$ plus a two-photon state $|\Psi\rangle$, where

$$
\begin{aligned}
|\Psi\rangle= & -\frac{i}{\hbar} \int_{-\infty}^{\infty} d t \hat{H}_{I}(t)|0\rangle \\
= & L \sum_{m, n} \int d \omega E_{p m} E_{c n} \operatorname{sinc}\left(\frac{\Delta k_{m n} L}{2}\right) \kappa_{m n}(-\omega, \omega) \\
& \times\left|\omega_{s m}-\omega, \omega_{a s n}+\omega\right\rangle .
\end{aligned}
$$

We consider that the modulated Stokes and anti-Stokes photons are detected by detectors $D_{s}$ and $D_{a s}$ [Fig. 1(a)] at time $t_{s}$ and $t_{a s}$, located with equal distance from the center of the source. The Stokes and anti-Stokes field annihilation operators in time-space domain are denoted by their Fourier transforms,

$$
\begin{gathered}
\hat{a}_{s}\left(z, t_{s}\right)=\frac{1}{\sqrt{2 \pi}} \sum_{m} \int d \omega \hat{a}_{s m}(\omega) e^{i\left[-k_{s m}(\omega) z-\left(\omega_{s m}+\omega\right) t_{s}\right]}, \\
\hat{a}_{a s}\left(z, t_{a s}\right)=\frac{1}{\sqrt{2 \pi}} \sum_{n} \int d \omega \hat{a}_{a s n}(\omega) e^{i\left[k_{a s n}(\omega) z-\left(\omega_{a s n}+\omega\right) t_{a s}\right]} .
\end{gathered}
$$

By following the procedure in [20,21], the two-photon amplitude at the output surfaces is then given by

$$
\begin{aligned}
\Psi\left(t_{s}, t_{a s}\right)= & \left\langle 0\left|\hat{a}_{s}\left(-\frac{L}{2}, t_{s}\right) \hat{a}_{a s}\left(\frac{L}{2}, t_{a s}\right)\right| \Psi\right\rangle \\
= & \frac{L}{2 \pi_{m, n}} \sum d \omega E_{p m} E_{c n} \Phi_{m n}(\omega) \\
& \times \kappa_{m n}(-\omega, \omega) e^{-i \omega_{s m} t_{s}} e^{-i \omega_{a s n} t_{a s}} e^{-i \omega\left(t_{a s}-t_{s}\right)},
\end{aligned}
$$

where the longitudinal detuning function is defined as

$$
\Phi_{m n}(\omega)=\operatorname{sinc}\left(\frac{\Delta k_{m n} L}{2}\right) e^{i\left[k_{s m}(-\omega)+k_{a s n}(\omega)\right] L / 2} .
$$

With the use of $\omega_{s m}=\omega_{p m}-\Delta \omega_{21}$ and $\omega_{a s n}=\omega_{c n}+\Delta \omega_{21}$, the biphoton wave function (9) can be rewritten as

$$
\begin{aligned}
\Psi\left(t_{s}, t_{a s}\right)= & \frac{L}{2 \pi} \sum_{m, n} \int d \omega \Phi_{m n}(\omega) \kappa_{m n}(-\omega, \omega) \\
& \times E_{p m} E_{c n} e^{-i \omega_{p m} t_{s}} e^{-i \omega_{c n} t_{a s}} e^{-i \omega\left(t_{a s}-t_{s}\right)},
\end{aligned}
$$

where without changing the physics, we have dropped the phase term $e^{i \Delta \omega_{21}\left(t_{s}-t_{a s}\right)}$. Equation (11) is our starting point to address how to (arbitrarily) shape a two-photon wave packet by modulating two input driving field profiles.

Before proceeding with the numerical simulations, it is instructive to look at the physics in some simple cases. We therefore concentrate on the cases where the biphoton bandwidth is mainly determined by phase matching Eq. (10) 
in the large OD limit $[18,20,26]$. In the simplest case, $\kappa_{m n}($ $-\omega, \omega)=\kappa$ in Eq. (11) is approximately taken to be a constant. We also assume that the pump-laser excitation is weak enough so that all the atomic population remains in the ground state $|1\rangle$. This ground-state approximation allows us to ignore the frequency dispersion of the Stokes photon which propagates nearly the speed of light in vacuum [20]. Since the anti-Stokes fields are operating in the EIT condition, they will experience slow light effect. Because spontaneously emitted slow anti-Stokes photons automatically match their coupling discrete frequency components in the multiple EIT channels, the group velocities associated with different anti-Stokes channels are the same and controlled by the average intensity of the coupling beam [27], i.e., $V_{g m}$ $=V_{g} \ll c$. Then for each FWM channel as shown in Fig. 1(b), there are $k_{s m}=k_{p m}-\left(\Delta_{21}-\omega\right) / c \simeq k_{p m}-\Delta_{21} / c$ and $k_{a s n}=k_{c n}$ $+\Delta_{21} / c+\omega / V_{g}$. Under the above approximations, we have $\Delta k_{m n} \simeq \omega / V_{g}$ and $k_{s m}+k_{a s n} \simeq k_{p m}+k_{c n}+\omega / V_{g}$. In addition, we do not take into account small Raman gain and EIT loss. Equation (10) can thus be simplified as

$$
\Phi_{m n}(\omega) \simeq \operatorname{sinc}\left(\frac{\omega L}{2 V_{g}}\right) e^{i\left(\omega L / 2 V_{g}\right)} e^{i\left(k_{p m}+k_{c n}\right) L / 2} .
$$

As a consequence, the biphoton waveform (11) becomes

$$
\begin{aligned}
\Psi\left(t_{s}, t_{a s}\right)= & \frac{\kappa L}{2 \pi} \int d \omega \operatorname{sinc}\left(\frac{\omega L}{2 V_{g}}\right) e^{i\left(\omega L / 2 V_{g}\right)} e^{-i \omega\left(t_{a s}-t_{s}\right)} \\
& \times \sum_{m} E_{p m} e^{-i\left(\omega_{p m} t_{s}-k_{p m} L / 2\right)} \sum_{n} E_{c n} e^{-i\left(\omega_{c n} t_{a s}-k_{c n} L / 2\right)} .
\end{aligned}
$$

With $k_{p m}=\omega_{p m} / c, k_{c n}=\omega_{c n} / c$, and Eqs. (1) and (2), the biphoton waveform reduces to

$$
\Psi\left(t_{s}, t_{a s}\right) \simeq-i \kappa V_{g} E_{p}^{(+)}\left(t_{s}-t_{0}\right) E_{c}^{(+)}\left(t_{a s}-t_{0}\right) \Pi\left(t_{a s}-t_{s}, L / V_{g}\right),
$$

where $t_{0}=L /(2 c)$. The rectangular function $\Pi\left(\tau, L / V_{g}\right)=1$ is defined within $\tau \in\left[0, L / V_{g}\right]$ and otherwise zero. Because a typical value of $L$ is about several centimeters, the small time delay of $t_{0}\left(\ll L / V_{g}\right)$ is negligible. This simplifies Eq. (14) as

$$
\Psi\left(t_{s}, t_{a s}\right) \simeq-i \kappa V_{g} E_{p}^{(+)}\left(t_{s}\right) E_{c}^{(+)}\left(t_{a s}\right) \Pi\left(t_{a s}-t_{s}, L / V_{g}\right) .
$$

It is obvious from Eq. (15) that the input pump and coupling field profiles have been mapped into the two-photon wave packet in time domain. Alternatively, this offers a way to shape the two-photon amplitude by manipulating input field profile(s). The two-photon temporal correlation is thus the module squared of Eq. (15) [and Eq. (14)],

$$
R\left(t_{s}, t_{a s}\right) \equiv\left|\Psi\left(t_{s}, t_{a s}\right)\right|^{2} \simeq\left|\frac{2 \kappa V_{g}}{c \varepsilon_{0}}\right|^{2} I_{p}\left(t_{s}\right) I_{c}\left(t_{a s}\right) \Pi\left(t_{a s}-t_{s}, \frac{L}{V_{g}}\right),
$$

where $I_{p}\left(t_{s}\right)$ and $I_{c}\left(t_{a s}\right)$ are pump and coupling intensity profiles. As seen from Eqs. (15) and (16), the two-photon amplitude and temporal correlation generally depend on both $t_{s}$ and $t_{a s}$ instead of only $t_{a s}-t_{s}$, which is different from the nonmodulation case. In the nonmodulation case with $\mathrm{cw}$ in- put pump and coupling fields, the system Hamiltonian is time independent in the laboratory reference frame so that photon pairs are generated at any time with equal probability; therefore the two-photon correlation only depends on the relative time delay. With modulation on the pump and coupling fields, the time translation symmetry of the Hamiltonian in Eq. (5) is broken down. As a result, the first-order input field correlation $E_{p}^{(+)}\left(t_{s}\right) E_{c}^{(+)}\left(t_{a s}\right)$ is mapped into the Stokes-anti-Stokes biphoton wave function in the time domain.

In the case where only one of the lasers (e.g., the pump laser) is modulated with a period of $T$, the time-averaged temporal correlation is evaluated as

$$
R(\tau)=\frac{1}{T} \int_{0}^{T} R\left(t_{s}, t_{s}+\tau\right) d t_{s} \sim \bar{I}_{p} I_{c} \Pi\left(\tau, \frac{L}{V_{g}}\right),
$$

where $\bar{I}_{p}$ is the average pump intensity. From Eq. (17) the averaged correlation shows no interference between different channels. To reveal the interference in the biphoton correlation function $R\left(t_{s}, t_{a s}\right)$, we can perform a conditional measurement. We can use the periodical modulation of the pump beam as a gate signal to trigger the coincidence detection of the Stokes and anti-Stokes photons. That is, detections at $D_{s}$ and $D_{a s}$ are conditioned upon at the gate signal from the modulation [28]. In this way, the time information, $t_{s}$ and $t_{a s}$, can be both obtained. When both pump and coupling fields are modulated, the pump-coupling intensity correlation is automatically mapped into the paired Stokes-anti-Stokes temporal correlation, as shown in Eq. (16). In such a case the time-averaged correlation becomes

$$
R(\tau) \sim C(\tau) \Pi\left(\tau, \frac{L}{V_{g}}\right),
$$

where $\quad C(\tau) \equiv \lim _{\Delta T \rightarrow \infty} \frac{1}{\Delta T} \int_{0}^{\Delta T} I_{p}(t) I_{c}(t+\tau) d t \quad$ is the timeaveraged pump-coupling correlation function.

\section{NUMERICAL SIMULATION}

To verify the validity of the theory, we perform numerical simulations based on Eq. (11) which includes small Raman gain, EIT loss, and exact line shapes of every FWM channel. We consider a cold ${ }^{85} \mathrm{Rb}$ atomic cloud prepared in a twodimensional (2D) magneto-optical trap (MOT) with the same energy-level configuration used in Ref. [18]: $|1\rangle=\mid 5 S_{1 / 2}$, $F=2\rangle, \quad|2\rangle=\left|5 S_{1 / 2}, F=3\right\rangle, \quad|3\rangle=\left|5 P_{1 / 2}, F=3\right\rangle, \quad$ and $\quad|4\rangle$ $=\left|5 P_{3 / 2}, F=3\right\rangle$. The dephasing rates are $\gamma_{13}=\gamma_{14}=\gamma_{24}=\gamma_{23}$ $=2 \pi \times 3 \mathrm{MHz}$ and $\gamma_{12}=0.001 \gamma_{13}$. The 2D MOT has an OD of about 50 on the anti-Stokes transition along the longitudinal direction and a length $L$ of about $1.5 \mathrm{~cm}$. The pump detuning is $\Delta_{p}=30 \gamma_{13}$. Rabi frequencies of free running (without modulation) pump and coupling lasers are $\Omega_{c}$ $=2.0 \gamma_{13}$ and $\Omega_{p}=1.0 \gamma_{13}$ which result in a biphoton bandwidth of $0.6 \mathrm{MHz}$.

Figure 2 shows two-photon correlation function $R\left(t_{s}, t_{s}+\tau\right)$ conditioned at $t_{s}=n T$ in which only the pump field amplitude is modulated with a sine wave, $\sin \left(\omega_{m} t\right)$, with a period of $T=2 \pi / \omega_{m}=1 \mu \mathrm{s}$. The dashed line shows correlation without modulation. The leading edge spikes are optical 


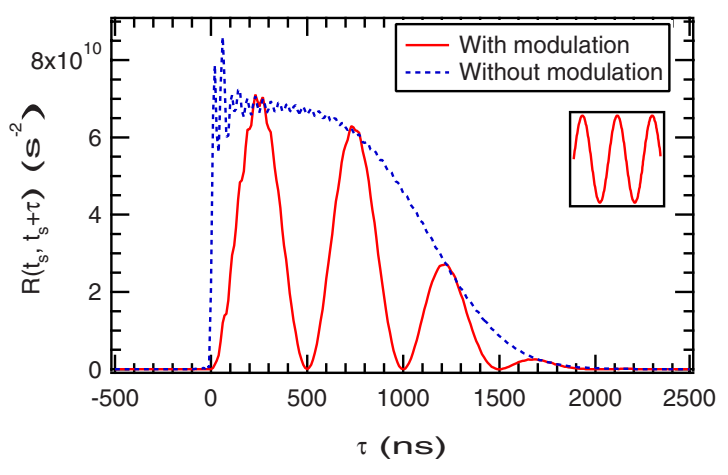

FIG. 2. (Color online) Conditional two-photon correlation function $R\left(t_{s}, t_{s}+\tau\right)$ at $t_{s}=n T$, where $T=1 \mu$ s is the modulation period and $n$ is an integer. The pump field amplitude is modulated with a sine wave (shown in the inset). The coupling laser operates in the cw mode. The dashed line shows correlation without modulation while the solid line with the modulation.

precursors resulting from both Stokes and anti-Stokes frequency components traveling at nearly the speed of light in vacuum and arriving at the detectors $D_{s}$ and $D_{a s}$ simultaneously [29]. The correlation time width of about $1.3 \mu \mathrm{s}$ is consistent with the group-delay time $L / V_{g}$ of the anti-Stokes photons and verifies the validity of Eq. (16). The solid line is the correlation with the modulation. The correlation modulation period of $T / 2=500 \mathrm{~ns}$ agrees with the period of the modulated pump intensity profile, $I_{p}(t) \propto \sin ^{2}\left(\omega_{m} t\right)$ $=\left[1-\cos \left(2 \omega_{m} t\right)\right] / 2$. Figure 3 shows the conditional twophoton correlation when the pump field amplitude is modulated with a rectangular wave with a modulation period of $1 \mu \mathrm{s}$. It is obvious that modulated square-wave patterns are revealed in the two-photon coincidences. Both Figs. 2 and 3 indicate that the modulation on the pump field profile can be recovered from the conditional biphoton coincidencecounting measurement. The conditional measurement here may offer a way to generate narrowband paired entangled photons on demand by timing the modulated pump (or coupling) field as a gate signal.

Figure 4 shows the time-averaged two-photon correlation function $R(\tau)$ when both the pump and coupling field ampli-

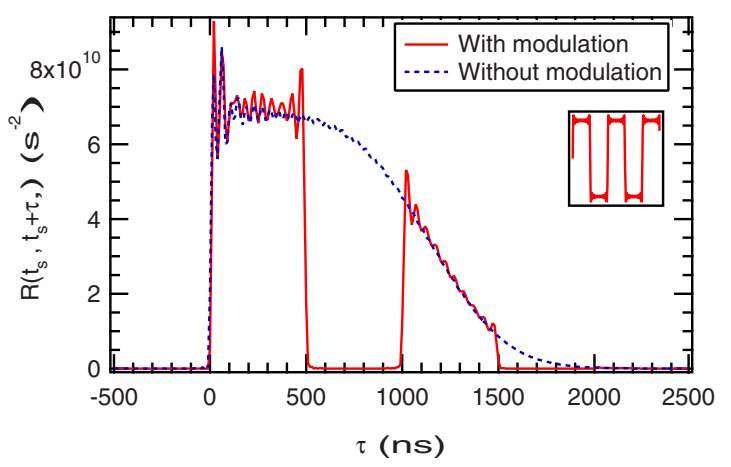

FIG. 3. (Color online) Conditional two-photon correlation function $R\left(t_{s}, t_{s}+\tau\right)$ at $t_{s}=n T$, where $T=1 \mu$ s is the modulation period and $n$ is an integer. The pump field amplitude is modulated with a rectangular wave (shown in the inset). The coupling laser works in the cw mode. The dashed line gives correlation without modulation while the solid line is the correlation with the modulation.

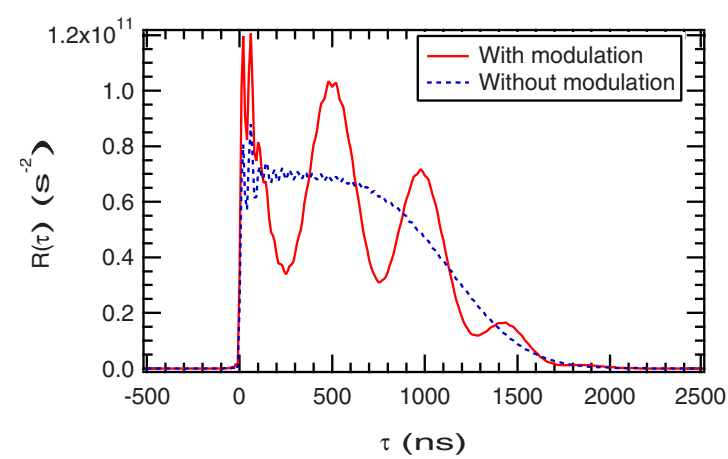

FIG. 4. (Color online) Time-averaged two-photon correlation function $R(\tau)$. Both pump and coupling field amplitudes are modulated with a sine wave. The dashed line shows correlation without modulation and the solid line gives the correlation with modulation.

tudes are (synchronously) modulated with the same sine wave. In such a case, the time-averaged input pump-coupling intensity correlation is $C(\tau) \propto(1 / T) \int_{0}^{T} \sin ^{2}\left[\omega_{m} t\right] \sin ^{2}\left[\omega_{m}(t\right.$ $+\tau)] d t=\left[2+\cos \left(2 \omega_{m} \tau\right)\right] / 8$ with a modulation period of $\pi / \omega_{m}$. The modulation period of the time-averaged twophoton correlation will coincide with this value, as demonstrated in Fig. 4 where both pump and coupling fields are modulated with a period of $T=1 \mu \mathrm{s}$. Several damped oscillation periods are clearly observable in the biphoton temporal coherence as shown in the simulation.

For simplicity, in the above numerical simulations we have only considered modulating the input field amplitude(s) to demonstrate the principle of the waveform shaping in the photon counting measurement. As shown in Eq. (14), both phase and amplitude modulations on the pump and coupling fields can be revealed in the biphoton quantum waveform. For example, one can apply frequency modulation (FM) to the driving fields and then measure the FM in the biphoton waveform using homodyne detection [7].

\section{DRIVING FIELDS WITH CONTINUOUS SPECTRUM}

The above theory may be generalized to the case where the pump and coupling fields have a continuous spectrum by replacing the sum with an integral. Following the similar procedure done in Sec. II, one has

$$
\begin{aligned}
\Psi\left(t_{s}, t_{a s}\right)= & \frac{L}{2 \pi} \int d \omega_{p} d \omega_{c} d \omega \Phi(\Delta k) \kappa\left(\omega_{p}, \omega_{c},-\omega, \omega\right) \\
& \times E_{p}\left(\omega_{p}\right) E_{c}\left(\omega_{c}\right) e^{-i \omega_{p} t_{s}} e^{-i \omega_{c} t_{a s}} e^{-i \omega\left(t_{a s}-t_{s}\right)},
\end{aligned}
$$

where $E_{p}\left(\omega_{p}\right)$ and $E_{c}\left(\omega_{c}\right)$ are spectral profiles of the pump and coupling fields, respectively. If $\Phi(\Delta k)$ and $\kappa$ are independent of $\omega_{p}$ and $\omega_{c}$, the two-photon wave function above can be approximated as $\Psi\left(t_{s}, t_{a s}\right) \sim E_{p}^{(+)}\left(t_{s}\right) E_{c}^{(+)}\left(t_{a s}\right) \psi\left(t_{a s}-t_{s}\right)$, similar to Eq. (14). In such a case, the linewidths of the pump and coupling lasers may not affect the time-averaged two-photon correlation shape if they are statistically independent. Further, the effects of finite driving-laser linewidths are not the same as the dephasing rate between the two ground levels. As an alternative approach, Eq. (19) can be used to study correlated photon generation using write-read techniques $[30,31]$. 


\section{SUMMARY}

In summary, we have discussed engineering biphoton waveforms with periodically modulated pump and coupling laser fields in a double- $\Lambda$ EIT atomic system. In the groupdelay regime where the biphoton coherence length is mainly determined by the phase-mismatching condition, we find that the input pump-coupling field-amplitude phase correlation can be mapped into the biphoton wave function within its coherence time window. This provides an engineering way to design and shape paired photons with controllable waveforms. The simulation parameters are taken from a 2D MOT with high OD, which is doable in the current laboratory [18]. We have also extended the theory into the case where two input pump and coupling fields have a continuous spectrum. For simplicity, we have focused on the collinear backward generation configuration, but the analysis presented here can be easily extended to other generation configurations such as forward [20] and right-angle [32] geometries. The proposed scheme may offer a way to generate narrowband biphotons on demand. It might be interesting to look at shaping biphoton wave packet in the Rabi-oscillation regime where the correlation profile is mainly determined by the third-order nonlinear susceptibility $[18,20,26]$, which is beyond the scope of this paper. It is expected that our results may be useful for quantum communication, quantum information processing, and engineering quantum state.

\section{ACKNOWLEDGMENTS}

The authors thank S. E. Harris, M. H. Rubin, G. Y. Yin, M. M. T. Loy, G. K. L. Wong, and P. Kolchin for helpful discussions. C.B. acknowledges support from the Defense Advanced Research Projects Agency, the U.S. Air Force Office of Scientific Research, and the U.S. Army Research Office. S.D. is grateful for the partial support from the Joyce M. Kuok Lasers and Photonics Laboratory. The work was support by the startup funding from the Department of Physics, the Hong Kong University of Science and Technology.
[1] M. Keller, B. Lange, K. Hayasaka, W. Lange, and H. Walther, Nature (London) 431, 1075 (2004).

[2] P. Kolchin, C. Belthangady, S. Du, G. Y. Yin, and S. E. Harris, Phys. Rev. Lett. 101, 103601 (2008).

[3] A. Pe'er, B. Dayan, A. A. Friesem, and Y. Silberberg, Phys. Rev. Lett. 94, 073601 (2005).

[4] S. Viciani, A. Zavatta, and M. Bellini, Phys. Rev. A 69, 053801 (2004).

[5] M. Hendrych, X. Shi, A. Valencia, and J. P. Torres, Phys. Rev. A 79, 023817 (2009).

[6] M. B. Nasr, S. Carrasco, B. E. A. Saleh, A. V. Sergienko, M. C. Teich, J. P. Torres, L. Torner, D. S. Hum, and M. M. Fejer, Phys. Rev. Lett. 100, 183601 (2008).

[7] S. E. Harris, Phys. Rev. Lett. 98, 063602 (2007).

[8] A. Valencia, A. Cere, X. Shi, G. Molina-Terriza, and J. P. Torres, Phys. Rev. Lett. 99, 243601 (2007).

[9] T. E. Keller and M. H. Rubin, Phys. Rev. A 56, 1534 (1997).

[10] Y. M. Mikhailova, P. A. Volkov, and M. V. Fedorov, Phys. Rev. A 78, 062327 (2008).

[11] V. Balić, D. A. Braje, P. Kolchin, G. Y. Yin, and S. E. Harris, Phys. Rev. Lett. 94, 183601 (2005).

[12] J. K. Thompson, J. Simon, H. Loh, and V. Vuletic, Science 313, 74 (2006).

[13] P. Kolchin, S. Du, C. Belthangady, G. Y. Yin, and S. E. Harris, Phys. Rev. Lett. 97, 113602 (2006).

[14] S. Du, J.-M. Wen, M. H. Rubin, and G. Y. Yin, Phys. Rev. Lett. 98, 053601 (2007).

[15] Q.-F. Chen, B.-S. Shi, M. Feng, Y.-S. Zhang, and G.-C. Guo, Opt. Express 16, 21708 (2008).
[16] S. E. Harris, Phys. Rev. A 78, 021807(R) (2008).

[17] C. Belthangady, S. Du, C.-S. Chuu, G. Y. Yin, and S. E. Harris (unpublished).

[18] S. Du, P. Kolchin, C. Belthangady, G. Y. Yin, and S. E. Harris, Phys. Rev. Lett. 100, 183603 (2008).

[19] S. E. Harris, Phys. Today 50 (7), 36 (1997).

[20] S. Du, J.-M. Wen, and M. H. Rubin, J. Opt. Soc. Am. B 25, C98 (2008).

[21] J.-M. Wen and M. H. Rubin, Phys. Rev. A 74, 023808 (2006); 74, 023809 (2006).

[22] P. Kolchin, Phys. Rev. A 75, 033814 (2007).

[23] C. H. Raymond Ooi, Q. Sun, M. S. Zubairy, and M. O. Scully, Phys. Rev. A 75, 013820 (2007).

[24] J.-M. Wen, S. Du, Y. P. Zhang, M. Xiao, and M. H. Rubin, Phys. Rev. A 77, 033816 (2008).

[25] M. H. Rubin, D. N. Klyshko, Y. H. Shih, and A. V. Sergienko, Phys. Rev. A 50, 5122 (1994).

[26] J.-M. Wen, S. Du, and M. H. Rubin, Phys. Rev. A 76, 013825 (2007).

[27] D. D. Yavuz, Phys. Rev. A 75, 031801(R) (2007).

[28] J. J. Thorn, M. S. Neel, V. W. Donato, G. S. Bergreen, R. E. Davies, and M. Beck, Am. J. Phys. 72, 1210 (2004).

[29] S. Du, C. Belthangady, P. Kolchin, G. Y. Yin, and S. E. Harris, Opt. Lett. 33, 2149 (2008)

[30] D. Matsukevich and A. Kuzmich, Science 306, 663 (2004).

[31] J. Laurat, H. de Riedmatten, D. Felinto, C. W. Chou, E. W. Schomburg, and H. J. Kimble, Opt. Express 14, 6912 (2006).

[32] S. Du, E. Oh, J.-M. Wen, and M. H. Rubin, Phys. Rev. A 76, 013803 (2007). 